\title{
Solute Effects on the Helix-Coil Transition
}

\author{
Oded Farago and Philip Pincus \\ Materials Research Laboratory, University of California, \\ Santa Barbara, California 93106
}

November 19, 2018

\begin{abstract}
We discuss the effects of the solvent composition on the helix-coil transition of a polypeptide chain. We use a simple model to demonstrate that improving the hydrogen-bonding ability of the solvent can make the transition less cooperative, without affecting the transition temperature. This effect is very different from other solvent effects which primarily influence the melting transition rather than the cooperativity.
\end{abstract}

PACS 87.15.-v, 05.70.Fh, 61.41.+e 
Proteins and nucleic acids (DNA and RNA) are biopolymers which are involved in almost every biological process. These molecules are characterized by special hierarchical structures [1]: Each chain consists of a unique sequence of bases (DNA) or amino acids (proteins), called the primary structure, which determines its specific biological function. Short-range interactions between nearby monomers along the chain can lead to the formation of a three-dimensional structure, usually a helix or a planar sheet, which is called the secondary structure of the chain. The secondary structure is in large measure determined by the primary structure. However, it also depends on the environmental conditions that the polymer experiences like the temperature or the composition of the solvent in which the chain is embedded. It is well known, for instance, that upon increasing the temperature, the helix structure "melts" into a random coil structure [2]. Another example is the folding-unfolding transition taking place when proteins are stretched by external forces [3]. In this paper we study the effects of the solvent composition on the helix-coil transition with the emphasis on a particular effect occurring when hydrogen-bonding agents are added to the solvent. We present a simple model which quantifies this effect, and compare it to other solvent effects, as well as to the effect that temperature or externally applied force have on the transition. While we will mainly deal with helix-coil transition occurring in polypeptides, we believe that the model may also apply to the transition observed in DNA experiments, just as the temperature-induced transition in both systems is studied by means of the same model.

Polypeptides are chains of amino acids monomers linked to each other by covalent peptide bonds. Each monomer can exist either in a $\alpha$-helical $(h)$ or a coil $(c)$ state. In a helical domain hydrogen bonding occurs along the polypeptide backbone between monomers separated by approximately 4 units. These hydrogen bonds make the $h$ state energetically more favorable than the $c$ state. The $c$ state is entropically favorable because of the rotational degrees of freedom of the amino acids. The helical monomers at the boundaries between $h$ and $c$ domains represent a special case: These monomers lose their configurational entropy with no energy gain due to the formation of hydrogen bonds. The thermodynamic behavior of the chain can be described using the Zimm-Bragg model [ब, in which the free energy associated with a particular configuration (a sequence of $h$ and $c$ monomers) of the chain is given by

$$
F^{\mathrm{conf}}=N_{h} \Delta F+2 N_{d} \Delta W
$$


In the above equation $N_{h}$ denotes the number of monomers in the $h$ state, $N_{d}$ is the number of helical domains, $\Delta F$ is the excess free energy per monomer in the helical state, and $\Delta W$ is the cost in free energy to create a boundary separating $h$ and $c$ domains. The partition function is readily obtained using the transfer matrix approach which is also applied to solve the onedimensional Ising model [5]. In the Zimm-Bragg model, the transition matrix is given by

$$
M=\left(\begin{array}{cc}
M_{c \rightarrow c} & M_{c \rightarrow h} \\
M_{h \rightarrow c} & M_{h \rightarrow h}
\end{array}\right)=\left(\begin{array}{cc}
1 & \sqrt{\sigma} s \\
\sqrt{\sigma} & s
\end{array}\right),
$$

where

$$
s=\exp (-\Delta F / k T),
$$

and

$$
\sigma=\exp (-2 \Delta W / k T)
$$

are the so-called Zimm-Bragg parameters, $k$ is the Boltzmann constant, and $T$ is the temperature. At low temperatures $\Delta F<0(s>1)$ since the energy gain in having an amino acid at a $h$ state overcomes the entropy loss compared to the $c$ state. Therefore in the low $T$ regime most of the monomers are in the $h$ state. At high temperatures $s<1$, and the chain is mostly in the $c$ state. The helix-coil melting transition occurs when $s \simeq 1$. In contrast to the significant variation of $s$ with the temperature, the parameter $\sigma$ (also called the cooperativity parameter) has only weak dependence on $T$. For biopolymers $\sigma$ is typically very small in the range $10^{-3}-10^{-4}$. The smallness of $\sigma$ reflects the large free energy penalty in creating a helix-coil interface, and is the reason for the sharpness of the helix-coil transition (which, due to the one-dimensional nature of the system, is not a true phase transition).

To understand the helix-coil transition in a more quantitative manner, we write the free energy of a chain consisting of $N(N \gg 1)$ monomers:

$$
F \simeq-N k T \ln x_{1}
$$

where $x_{1}$ is the largest eigenvalue of the transfer matrix $M$ [Eq.(2)]. The eigenvalues of $M$ are the roots of the quadratic equation

$$
(1-x)(s-x)-s \sigma=0 .
$$

The largest root of this equation is equal to

$$
x_{1}=\frac{1}{2}\left[1+s+\sqrt{(1-s)^{2}+4 s \sigma}\right] .
$$




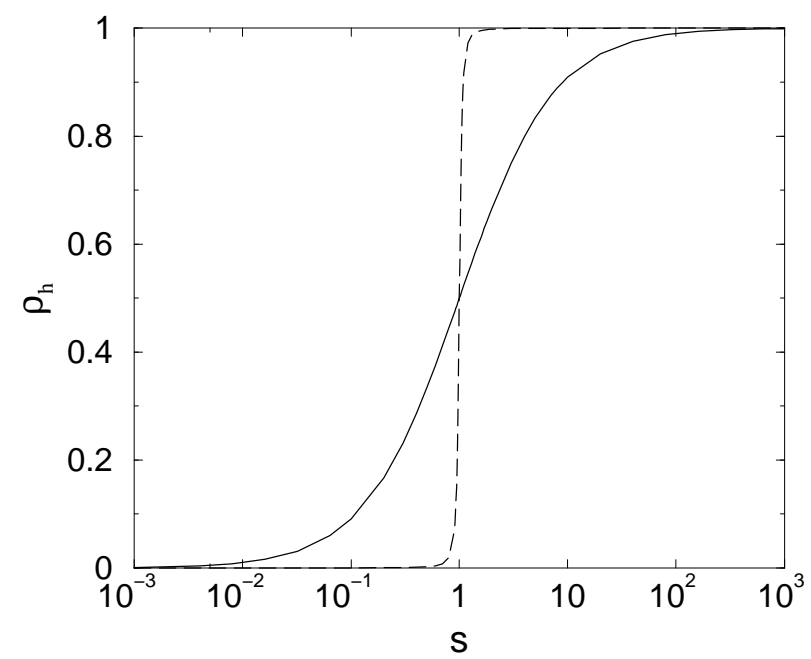

Figure 1: The fraction of monomers in the $h$ state, $\rho_{h} \equiv\left\langle N_{h}\right\rangle / N$, as a function of the Zimm-Bragg parameter $s$, for $\sigma=10^{-3}$ (dashed line) and $\sigma=1$ (solid line). The curves intersect at $s=1$, where $\rho_{h}=1 / 2$.

The average number $\left\langle N_{h}\right\rangle$ of monomers in the $h$ state is derived by differentiating the free energy [Eqs.(5) and (7)] with respect to $\Delta F=-k T \ln s$. We obtain

$$
\left\langle N_{h}\right\rangle=\frac{\partial F}{\partial(\Delta F)}=N\left[\frac{1}{2}+\frac{s-1}{2 \sqrt{(s-1)^{2}+4 s \sigma}}\right] .
$$

The dependence of $\rho_{h} \equiv\left\langle N_{h}\right\rangle / N$, the fraction of monomers in the $h$ state, on $s$ is plotted in Fig. 1, for $\sigma=10^{-3}$ and $\sigma=1$ (the non-cooperative case). We observe that $\rho_{h} \simeq 0$ for $s \ll 1$, while $\rho_{h} \simeq 1$ for $s \gg 1$. The crossover between these two regimes occur at $s \simeq 1$ (at $s=1$, the fraction of helical monomers is exactly $1 / 2$ ). The transition becomes sharper with decreasing $\sigma$. For small values of $\sigma$ the width of the transition regime scales as $\Delta s \sim \sigma^{1 / 2}$.

When dealing with the possible influence of the solvent composition on the transition we should account for two types of effects. Effects of the first type decrease the relative stability of the $h$ state over the $c$ state. Effects of the second type reduce the helix-coil interfacial free energy.

Within the first group, we include changes in the $p \mathrm{H}$ or the ionic strength of the solution that destabilize the $h$ state [6]. One can incorporate these 
changes in the Zimm-Bragg model by a proper redefinition of the parameter $s$, which now becomes a function of both the temperature and the solution conditions. As a result of the change in the solvent conditions, the transition (melting) temperature shifts, and it is now defined by $s^{*}\left(T_{\mathrm{m}}\right)=1$ with $s^{*}$ being the solvent-dependent parameter.

It is interesting to note that the force-induced transition, observed in recent stretching experiments of DNA [0], has been also explained by the Zimm-Bragg theory with a rescaled (in this case, a force-dependent) parameter $s[8]$. The effect of externally applied force on the transition is usually studied in the fixed-force $(f)$ ensemble. On a mean-field level (which turns out to provide a rather good description of the experimentally measured elastic behavior), the elastic free energy is given by

$$
F^{\mathrm{el}}(f)=N_{h} E_{h}^{\mathrm{el}}(f)+\left(N-N_{h}\right) E_{c}^{\mathrm{el}}(f),
$$

where $E_{h}^{\mathrm{el}}(f)$ and $E_{c}^{\mathrm{el}}(f)$ denote, respectively, the elastic free energy per monomer in the pure $h$ and $c$ states (i.e., when all the monomers are in the same state). The elastic behavior of the pure phases is usually described by one of the generic polymer models like the freely-jointed or worm-like chain models [9]. Within these models, the elastic energy per monomer is given by

$$
E^{\mathrm{el}}(f)=-\frac{1}{N} \int_{0}^{f} R\left(f^{\prime}\right) d f^{\prime}
$$

where $R(f)$ is the (mean) end-to-end separation of the chain. The transfer matrix corresponding to the sum of configurational (11) and elastic (8) free energies is

$$
M=\left(\begin{array}{cc}
M_{c \rightarrow c} & M_{c \rightarrow h} \\
M_{h \rightarrow c} & M_{h \rightarrow h}
\end{array}\right)=e^{-E_{c}^{\mathrm{el}}(f) / k T}\left(\begin{array}{cc}
1 & \sqrt{\sigma} s e^{\left[E_{c}^{\mathrm{el}}(f)-E_{h}^{\mathrm{el}}(f)\right] / k T} \\
\sqrt{\sigma} & s e^{\left[E_{c}^{\mathrm{el}}(f)-E_{h}^{\mathrm{el}}(f)\right] / k T}
\end{array}\right),
$$

and by comparison with the Zimm-Bragg transfer matrix (2) we readily conclude that $s^{*}(f)=s e^{\left[E_{c}^{\mathrm{el}}(f)-E_{h}^{\mathrm{el}}(f)\right] / k T}$.

Let us consider the change in the behavior of polypeptide chains that occurs when the hydrogen-bonding ability of the solvent is improved. One may consider this as another example of a solvent effect of the first type. In alcohol environment, for instance, the hydroxide groups tend to form hydrogen bonds with the coil monomers, thus increasing the relative stability of the latter compared to the helical monomers. This effect is enhanced if the 
hydrogen-bonding ability of the solvent with the $c$ monomers is improved, e.g., if water is added to the solution [10]. However, one may consider the following different scenario which serves as an example of a solvent effect of the second type. Suppose the polypeptide chain is dissolved in an aqueous solution, and most of the coil monomers are involved in hydrogen bonds with the water molecules. Let us assume now that the hydrogen-bonding ability of the solvent is improved by adding molecules which prefer to bind to the terminal monomers of the helical domains. The high affinity of the new hydrogen-bonding agents (which we shall call "impurities") for the terminal $h$ monomers leads to the formation of new hydrogen bonds at the interfaces between $h$ and $c$ domains. To introduce the effect of these additional hydrogen bonds, we need to add a term to the configurational free energy [Eq.(1)]

$$
F^{\mathrm{conf}}=N_{h} \Delta F+2 N_{d} \Delta W+\mu N_{\mathrm{i}},
$$

where $\mu$ is the chemical potential per impurity molecule attached to the chain at the interface between $h$ and $c$ domains, while $N_{\mathrm{i}}\left(N_{\mathrm{i}} \leq 2 N_{d}\right)$ denotes the number of such impurities [11]. It is useful to write

$$
\mu=\Delta \tilde{W}-\Delta W-k T \ln \varphi
$$

where $\Delta \tilde{W}$ is the free energy per "impure" interface with an additional hydrogen bond (replacing $\Delta W$, the free energy of a "pure" interface), while $\varphi \equiv N \xi^{3} / V$ is the concentration of the free impurity molecules in the solvent (with $\xi$, the "thermal wavelength"). The last term on the r.h.s of Eq.(10) represents the loss of mixing entropy of the impurity molecules connected to the chain compared to the free impurities (assuming that $\varphi$ is sufficiently low). All the other contributions to the free energy of the impure interfaces, for instance the binding energy between the impurities and the chain, are included in $\Delta \tilde{W}$.

In order to find the free energy corresponding to Eq.(9) we define two new states $h^{*}$ and $c^{*}$ representing, respectively, the terminal ("C-end") monomer of a helical or a coil domain followed by an impure interface. We can then apply the transfer matrix approach, where in the present case the transfer matrix is the $4 \times 4$ matrix

$$
M=\left(\begin{array}{llll}
M_{c \rightarrow c} & M_{c \rightarrow h} & M_{c \rightarrow c^{*}} & M_{c \rightarrow h *} \\
M_{h \rightarrow c} & M_{h \rightarrow h} & M_{h \rightarrow c^{*}} & M_{h \rightarrow h^{*}} \\
M_{c^{*} \rightarrow c} & M_{c^{*} \rightarrow h} & M_{c^{*} \rightarrow c^{*}} & M_{c^{*} \rightarrow h^{*}} \\
M_{h^{*} \rightarrow c} & M_{h^{*} \rightarrow h} & M_{h^{*} \rightarrow c^{*}} & M_{h^{*} \rightarrow h^{*}}
\end{array}\right)=\left(\begin{array}{cccc}
1 & \sqrt{\sigma} s & 1 & \sqrt{\sigma} s \\
\sqrt{\sigma} & s & \sqrt{\sigma} & s \\
0 & \sqrt{\tilde{\sigma}} s & 0 & \sqrt{\tilde{\sigma}} s \\
\sqrt{\tilde{\sigma}} & 0 & \sqrt{\tilde{\sigma}} & 0
\end{array}\right),
$$


with

$$
\tilde{\sigma}=\exp [-2(\Delta \tilde{W}-k T \ln \varphi) / k T]=\exp (-2 \Delta \tilde{W} / k T) \varphi^{2}
$$

The eigenvalues of the transfer matrix (11) are the roots of the polynomial equation

$$
x^{2}[(1-x)(s-x)-s(\sigma+2 \sqrt{\sigma \tilde{\sigma}}+\tilde{\sigma})]=0 .
$$

Although this is a fourth order equation, the largest root (which is relevant to the thermodynamic behavior) comes from the quadratic equation defined in brackets in Eq.(12)

$$
(1-x)(s-x)-s(\sigma+2 \sqrt{\sigma \tilde{\sigma}}+\tilde{\sigma})=0 .
$$

This equation is similar to the quadratic equation of the Zimm-Bragg model [Eq.(6)], with the cooperativity parameter $\sigma$ replaced by

$$
\sqrt{\sigma^{*}}=\sqrt{\sigma}+\sqrt{\tilde{\sigma}}=\sqrt{\sigma}+\exp (-\Delta \tilde{W} / k T) \varphi .
$$

Our model, therefore, yields a description of the helix-coil transition similar to the Zimm-Bragg model, but with a larger cooperativity parameter. We find that $\sigma^{*}>\sigma$ even if the chemical potential $\mu$ in Eqs.(9) and (10) is positive, i.e., if the binding of the impurities to the chain increases the free energy of the system. This result is due to the simple fact that some of the helix-coil interfaces will have impurities even when $\mu>0$ and, consequently, the free energy cost per interface will decrease (compared to the case when we had no impurities at all, $\varphi=0$ ). In the "neutral" case $\mu=0$, for instance, half of the hydrogen-bonding impurities will be attached to the chain, and from Eqs.(4), (10), and (13) we find that $\sigma^{*}=4 \sigma$. We thus conclude that the helix-coil transition becomes broader in the presence of the hydrogenbonding impurities, but quite unusually, the melting temperature (which is associated with the parameter $s$ ) is not predicted to change. This effect is, therefore, markedly different from the other ("first type") solvent effects, as well as from the effect of externally applied force on the transition.

To summarize, we propose a simple description for the complicated dependence of the helix-coil transition on the solvent character. We suggest that solvent effects can be characterized by their impact on the Zimm-Bragg parameters. Accordingly, we can broadly classify them into two types: Solvent effects of the first type are associated with a shift in the position of the 
helix-coil transition, and the rescaling of the parameter $s$. Solvent effects of the second type reduce the cooperativity of the transition, i.e., increase the parameter $\sigma$. The amount by which $\sigma$ grows depends on the binding free energy of the impurities, and is proportional to their concentration in the solution. This, however, applies only to low concentrations. At larger densities it is necessary to include higher virial coefficients in the mixing-entropy term [Eq.10)] to describe the interactions between the impurities.

We thank the referee for his very useful remarks, and M. C. Williams for bringing our attention to his experimental work on this subject. We also

acknowledge discussions with K. Plaxco, J. Israelachvili and T. Deming. The work was supported by the MRL Program of the National Science Foundation under Award No. DMR00-80034.

\section{References}

[1] C. Branden and J. Tooze, Introduction to Protein Structure (Graland Publishing, New York, 1991).

[2] D. Poland and H. A. Scheraga, Theory of Helix-Coil Transition in Biopolymers (Academic Press, New York, 1970); C. R. Cantor and P. R. Schimmel, Biophysical Chemistry (Freeman, New York, 1980); A. Y. Grosberg and A. R. Khokhlov, Statistical Physics of Macromolecules (AIP Press, New York, 1994).

[3] M. Rief, F. Oesterhelt, B. Heymann, and H. E. Gaub, Science 275, 1295 (1997); M. S. Z. Kellermayer, S. B. Smith, H. L. Granzier, and C. Bustamante, Science 276, 1112 (1997); F. Oesterhelt, M. Reif, and H. E. Gaub, New. J. Phys. 1, 6.1 (1999).

[4] B. H. Zimm and J. K. Bragg, J. Chem Phys. 31, 526 (1959).

[5] N. Goldenfeld, Lectures on Phase Transitions and the Renormalization Group (Addision-Wesley, New York, 1992).

[6] J. Bond, C. Anderson, and M. T. Record Jr., Biophys. J. 67, 825 (1994); M. C. Williams, J. R. Wenner, I. Rouzina, and V. A. Bloomfield, Biophys. J. 80, 874 (2001); I. Rouzina and V. A. Bloomfield, Biophys. J. 80, 894 (2001). 
[7] See e.g., P. Cluzel, A. Lebrun, C. Heller, R. Lavery, J. L. Viovy, D. Chatenay, and F. Caron, Science 271, 792 (1996); S. B. Smith, Y. Cui, and C. Bustamante, Science 271, 795 (1996); M. C. Williams, J. R. Wenner, I. Rouzina, and V. A. Bloomfield, Biophys. J. 80, 1932 (2001).

[8] A. Ahsan, J. Rudnick, and R. Bruinsma, Biophys. J. 74, 132 (1998); I. Rouzina and V. A. Bloomfield, Biophys. J. 80, 882 (2001). See also the following references that deal with stretching of polypeptides using the same theoretical approach: A. Buhot and A. Halperin, Phys. Rev. Lett. 84, 2160 (2000); M. N. Tamashiro and P. Pincus, Phys. Rev. E 63, 021909 (2001).

[9] M. Doi and S. F. Edwards, The Theory of Polymer Dynamics (Oxford University Press, Oxford, 1988). For slightly modified versions of these models see, e.g., J. F. Marko and E. D. Siggia, Macromol. 28, 8759 (1995), or the paper by Smith, Cui and Bustamante in Ref. [7].

[10] I. V. Soman, A. Karimi, and D. A. Case, Biopolymers 31, 1351 (1991); C. A. Schiffer and V. Dötsch, Curr. Opin. Biotechnol. 7, 428 (1996); P. Doruker and I. Bahar, Biophys. J. 72, 2445 (1997); L. D Barron, L. Hecht, and G. Wilson, Biochemistry. 36, 13143 (1997).

[11] In Eq.(9) we assume that all the new hydrogen bonds are formed at the interfaces between helix and coil domains. This assumption applies to the case when the free energy gain in creating such a new bond is much larger than the free energy gain in replacing an existing bond between a coil monomer and a water molecule, with a bond between a coil monomer and impurity. 